\title{
Biosynthesis of cannabinoid precursor olivetolic acid by overcoming rate-limiting steps in genetically engineered Yarrowia lipolytica
}

\author{
Jingbo $\mathrm{Ma}^{1}$, Yang $\mathrm{Gu}^{1,2^{*}}$ and Peng $\mathrm{Xu} \mathrm{u}^{1,3^{*}}$ \\ ${ }^{1}$ Department of Chemical, Biochemical and Environmental Engineering, University of \\ Maryland, Baltimore County, Baltimore, MD 21250 \\ ${ }^{2}$ School of Food Science and Pharmaceutical Engineering, Nanjing Normal University, \\ Nanjing, China \\ ${ }^{3}$ Department of Chemical Engineering, Guangdong Technion-Israel Institute of Technology, \\ Shantou, Guangdong 515063
}

\footnotetext{
"Corresponding authors Tel: + 86-0754-88077088. E-mail: guyang@nnu.edu.cn (YG) and peng.xu@gtiit.edu.cn (PX).
} 


\begin{abstract}
Natural products acting on our central nervous systems are in utmost demand to fight against pain and mental disorders. Cannabinoids (CBDs) are proven neuroactive agents to treat anxiety, depression, chronic pain diseases, seizure, strokes and neurological disorders. The scarcity of the hemp-sourced CBD products and the prohibitive manufacturing cost limit the wide application of CBDs. Yeast metabolic engineering offers the flexibility to meet the ever-increasing market demand. In this work, we took a retrosynthetic approach and sequentially identified the rate-limiting steps to improve the biosynthesis of the CBD precursor olivetolic acid (OLA) in Yarrowia lipolytica. We debottlenecked the critical enzymatic steps to overcome the supply of hexanoyl-CoA, malonyl-CoA, acetyl-CoA, NADPH and ATPS to redirect carbon flux toward OLA. Implementation of these strategies led to an 83-fold increase in OLA titer in shaking flask experiment. This work may serve as a baseline for engineering CBD biosynthesis in oleaginous yeast species.
\end{abstract}

Keywords: Metabolic Engineering, Oleaginous yeast, Cannabinoids, Olivetolic acid, Natural Products, Biosynthesis 


\section{Introduction}

Cannabinoids (CBDs) are a large family of neurological active compounds. Cell biologists have identified multiple cannabinoids receptor proteins in human brain and found CBDs or their derivatives played an important role to regulate human cognitive and emotional functions ${ }^{1,2}$. Recent pharmaceutical and clinical studies have proved that CBDs can be used to treat anxiety, depression, aging-related muscle/joint pain, seizure, stroke and cardiovascular diseases et $a l^{3,4}$. Almost all CBDs are sourced from farm-harvested hemp or Cannabis sativa-related species. Recent molecular breeding has resulted in special hemp cultivars which synthesize less tetrahydrocannabinol (THC) -- the principle psychoactive constituent of cannabis (Marijuana) ${ }^{5}$. CBD oils free of THCs will not cause human addiction. FDA and World Health Organization (WHO) have legally approved the use of CBDs as an essential drug ingredient to treat neurological disorders. Recently, a growing number of nations have lifted the restrictions and approved the use of THC-free CBDs as a consumer chemical in human food, drink, cosmetics and nutraceutical industry. For example, hemp-derived $\mathrm{CBD}$ oil (free of $\mathrm{THC}$ ) has been infused with human lotion, confectionary (gummies or cookies) or cigarette products. The overall market size of CBD oil as consumer chemicals is estimated to be $\$ 25$ billion in 2025 (assuming 50 Million people will use CBDs as a consumer chemical daily, average dosage $50 \mathrm{~g} /$ people/year or $2 \mathrm{mg} / \mathrm{kg}$-body-weight/day). Current crude CBD oil (45\% strength) is sold at about $\$ 3,000 / \mathrm{kg}$ and pure CBD oil is sold at $\$ 0.01$ per milligram. CBD market is projected to increase $10 \%-15 \%$ annually, the current hemp planting and agricultural technology cannot meet this rapid market demand. The pandemics of Covid-19 has severely disrupted our supply chain of food, pharmaceuticals and consumer chemicals, driving us to seek alternate solutions to manufacture essential drugs and safeguard our well-being ${ }^{6}$. There is an urgent need to develop alternate route to fill the CBD supply chain.

Microbial metabolic engineering is considered as the enabling technology to mitigate environmental concerns and address the scarcity of resource limitation challenges ${ }^{7,8}$. Compared to plant-based production system, microbes have a number of advantages, including less dependence on arable land or climate changes, ease of genetic manipulation 
and large-scale production, as well as robust growth and high conversion rate across a wide range of low-cost renewable raw materials ${ }^{6,9}$. Therefore, genetically modified microbes have been widely used to produce fuels, commodity chemicals and nutraceuticals ${ }^{10}$. With our increased knowledge of cellular physiology and understanding of molecular genetics toward higher and complex organisms, there is a growing interest to develop novel cellular chassis that may overcome the constraints of common host (E. coli and S. cerevisiae) ${ }^{11}$. For example, Yarrowia lipolytica, is characterized as a generally regarded as safe (GRAS) oleaginous yeast, has been recently modified to produce an arrange of value-added natural products, including resveratrol ${ }^{12}$, squalene ${ }^{13}$, flavonoids ${ }^{14}$, artemisinin ${ }^{15}$ and violacein ${ }^{16}$ et al. The distinct oil-accumulating (hydrophobic) environment, abundance of intracellular membrane surface, and the compartmentalization of biosynthetic pathways in Y. lipolytica provide the ideal microenvironment for the catalytic functions of enzymes with stereo- or regio-selectivity ${ }^{11}$. This property is extremely important for the functional expression of P450 enzymes with site-specific hydroxylation or peroxidation reactions. CBD biosynthetic pathway involves polyketide synthase, mevalonate pathway and prenyltransferase, which posts a significant challenge for efficient biosynthesis in common host organism ${ }^{17} . Y$. lipolytica is reported to accommodate strong flux for acetyl-CoA, malonyl-CoA and HMG-CoA. Naturally, Y. lipolytica could be a promising host to produce CBDs and their derivatives. In this work, we explored the possibility to use oleaginous yeast $Y$. lipoltyica as the host to synthesize olivetolic acid, a universal precursor to synthesize CBDs. We overcome a number of critical pathway bottlenecks to unlock the potential of $Y$. lipolytica to synthesize olivetolic acids. These strategies, when combined, lead to a more than 83-fold increase in olivetolic acid production. This preliminary result may provide a baseline for us to develop CBD-producing oleaginous yeast cell factories.

\section{Material and Methods}

\subsection{Strains, plasmids, primers, and chemicals}

All strains of engineered $Y$. lipolytica, including the genotypes, recombinant plasmids, and primers have been listed in Supplementary Table 1 and 2. Olivetolic acid was purchased 
from Santa Cruz Biotechnology. All other chemicals were obtained from Sigma-Aldrich and Fisher Scientific. Heterologous synthetic genes including genes CSOLS, CsOAC, CSAAE1, CSAAE3, PpLvaE, SeACS ${ }^{\mathrm{L} 641 \mathrm{P}}$ and MCMAE2 were codon-optimized using the online IDT Codon Optimization Tool and then ordered from GENEWIZ (Suzhou, China). The synthetic gene fragments were assembled with the New England Biolab Gibson Assembly kits, with the pYaliBrick vector as plasmid backbone. DNA sequences were verified by Sanger sequencing (QuintaraBio).

\subsection{Shake flask cultivations and $\mathrm{pH}$ control}

For performing shake flask cultivations, seed culture was carried out in the shaking tube with $2 \mathrm{~mL}$ seed culture medium at $30{ }^{\circ} \mathrm{C}$ and 250 r.p.m. for $48 \mathrm{~h}$. Then, $0.6 \mathrm{~mL}$ of seed culture was inoculated into the $250 \mathrm{~mL}$ flask containing $30 \mathrm{~mL}$ of fermentation medium and grown under the conditions of $30^{\circ} \mathrm{C}$ and 250 r.p.m. for 96 h. One milliliter of cell suspension was sampled every $24 \mathrm{~h}$ for $\mathrm{OD}_{600}$ and desired metabolite measurement.

Seed culture medium used in this study included the yeast complete synthetic media regular media (CSM, containing glucose $20.0 \mathrm{~g} / \mathrm{L}$, yeast nitrogen base without ammonium sulfate $1.7 \mathrm{~g} / \mathrm{L}$, ammonium sulfate $5.0 \mathrm{~g} / \mathrm{L}$, and CSM-Leu $0.74 \mathrm{~g} / \mathrm{L}$ ) and complex medium (YPD, containing glucose $20.0 \mathrm{~g} / \mathrm{L}$, yeast extract $10.0 \mathrm{~g} / \mathrm{L}$, and peptone $20.0 \mathrm{~g} / \mathrm{L}$ ). Fermentation medium used in this study contained the yeast complete synthetic media regular media (CSM, containing glucose $40.0 \mathrm{~g} / \mathrm{L}$, yeast nitrogen base without ammonium sulfate $1.7 \mathrm{~g} / \mathrm{L}$, ammonium sulfate $1.1 \mathrm{~g} / \mathrm{L}$, and CSM-Leu $0.74 \mathrm{~g} / \mathrm{L}$ ) and complex medium (YPD, containing glucose $40.0 \mathrm{~g} / \mathrm{L}$, yeast extract $10.0 \mathrm{~g} / \mathrm{L}$, and peptone $20.0 \mathrm{~g} / \mathrm{L}$ ). To control the $\mathrm{pH}, 20 \mathrm{mM}$ phosphate buffer saline (PBS, $\mathrm{Na}_{2} \mathrm{HPO}_{4}-\mathrm{NaH}_{2} \mathrm{PO}_{4}$ ) or $20 \mathrm{~g} / \mathrm{L} \mathrm{CaCO}$ was used, respectively.

\subsection{Yeast transformation and screening of high-producing strains}

The standard protocols of Y. lipolytica transformation by the lithium acetate method

were described as previously reported ${ }^{16,18}$. In brief, one milliliter cells was harvested during the exponential growth phase $(16-24 \mathrm{~h}$ ) from $2 \mathrm{~mL}$ YPD medium (yeast extract $10 \mathrm{~g} / \mathrm{L}$, peptone $20 \mathrm{~g} / \mathrm{L}$, and glucose $20 \mathrm{~g} / \mathrm{L}$ ) in the 14-mL shake tube, and washed twice with 100 
$\mathrm{mM}$ phosphate buffer ( $\mathrm{pH}$ 7.0). Freshly cultivated yeast colony lawns picked from overnight-grown YPD plates could also be used for genetic transformation. Then, cells were resuspended in $105 \mu \mathrm{L}$ transformation solution, containing $90 \mathrm{uL}$ 50\% PEG4000, $5 \mu \mathrm{L}$ lithium acetate (2M), $5 \mu \mathrm{L}$ boiled single stand DNA (salmon sperm, denatured) and $5 \mu \mathrm{L}$ DNA products (including 200-500 ng of plasmids, linearized plasmids or DNA fragments), and incubated at $39{ }^{\circ} \mathrm{C}$ for $1 \mathrm{~h}$, then spread on selected plates. It should be noted that the transformation mixtures needed to be vortexed for 15 seconds every 15 minutes during the process of $39{ }^{\circ} \mathrm{C}$ incubation. The selected markers, including leucine, uracil and hygromycin, were used in this study. All engineered strains after genetic transformation were undergone PCR screening using the GoTaq Green PCR kits, and the strain containing the correct gene fragment was selected to perform shaker flask cultivation. For shaking tube cultivations, 100 $\mu \mathrm{L}$ seed cultures were inoculated into $5 \mathrm{~mL}$ fermentation media in a $50 \mathrm{~mL}$ tube.

\subsection{Single-gene and multi-genes expression vectors construction}

In this work, the YaliBrick plasmid pYLXP' was used as the expression vector ${ }^{19}$. The process of plasmid constructions followed the YaliBrick gene assembly platforms ${ }^{16}$. In brief, recombinant plasmids of $\mathrm{PYLXP}-x x$ (a single gene) were built by Gibson Assembly of linearized PYLXP' (digested by SnaBI and Kpnl) and the appropriate PCR-amplified or synthetic DNA fragments. Multi-genes expression plasmids were constructed based on restriction enzyme subcloning with the isocaudamers Avrll and Nhel. All genes were respectively expressed by the TEF promoter with intron sequence and XPR2 terminator, and the modified DNA fragments and plasmids were sequence-verified by Sanger sequencing (Quintarabio).

\subsection{Gene knockout}

A marker-free gene knockout method based on Cre-lox recombination system was used as previously reported ${ }^{16,20}$. For performing gene knockout, the upstream and downstream sequences (both $1000 \mathrm{bp)} \mathrm{flanking} \mathrm{the} \mathrm{deletion} \mathrm{targets} \mathrm{were} \mathrm{PCR-amplified.}$ These two fragments, the loxP-Ura/Hyr-loxP cassette (digested from plasmid pYLXP'-loxP-Ura/Hyr by Avrll and sall), and the gel-purified plasmid backbone of 
pYLXP'(linearized by Avrll and sall) were joined by Gibson Assembly, giving the knockout plasmids pYLXP'-loxP-Ura/Hyr-xx ( $x x$ is the deletion target). Next, the knockout plasmids were sequence-verified by Quintarabio. Then, the gene knockout cassettes were PCR-amplified from the knockout plasmids pYLXP'-loxP-Ura/Hyr-xx, and further transformed into Y. lipolytica. The positive transformants were determined by colony PCR. Knockout strains were built on top of the Ku70-deficient strains (Po1f background). Subsequently, plasmid PYLXP'-Cre was introduced into the positive transformants and promoted the recombination of IoxP sites, which recycle the selected marker. Finally, the intracellular plasmid pYLXP'-Cre was evicted by incubation at $30^{\circ} \mathrm{C}$ in YPD media for $48 \mathrm{~h}$. Here, Ura is the uracil marker, and Hyr is hygromycin marker.

\subsection{Genomic integration of desired genes}

In this work, genomic integration of desired genes was performed in two different ways: site-specific genomic integration plasmids or application of $\mathrm{pBR}$ docking platform by linearizing the plasmid pYLXP' with digested enzyme Notl. Here, we constructed two genomic integration plasmids pURLA and pURLB, corresponding to the $K u 70$ and YALIOC05907g (encoding a hypothetical protein conserved in the Yarrowia clade) genomic sites, respectively. The procedure of using these two plasmids was similar as that of gene knockout protocol. The method of constructing integration plasmids was described in previous work. The application of $\mathrm{pBR}$ docking platform was achieved by linearizing the plasmid pYLXP' with Not/ restriction enzyme digestion.

\subsection{HPLC quantification and LC-MS characterization of olivetolic acid}

Cell densities were monitored by measuring the optical density at $600 \mathrm{~nm}\left(\mathrm{OD}_{600}\right)$. The concentrations of olivetolic acid were measured by high-performance liquid chromatography (HPLC) through Agilent HPLC 1220. In detail, olivetolic acid was measured at $270 \mathrm{~nm}$ under $40{ }^{\circ} \mathrm{C}$ (column oven temperature) with a mobile phase containing $60 \%(\mathrm{v} / \mathrm{v})$ methanol in water at a flow rate of $0.4 \mathrm{~mL} / \mathrm{min}$ equipped with a ZORBAX Eclipse Plus C18 column $(4.6 \times$ $100 \mathrm{~mm}, 3.5 \mu \mathrm{m}$, Agilent) and the VWD detector.

To quantify the concentration of olivetolic acid, $0.5 \mathrm{~mL}$ whole cell sample with both 
cell pellet and liquid culture was taken. Subsequently, samples were treated with 2 U/OD zymolyase ( $2 \mathrm{~h}, 30^{\circ} \mathrm{C}$ with shaking at $\left.1000 \mathrm{rpm}\right)$, and then cell suspensions were added with $20 \%(\mathrm{w} / \mathrm{v})$ glass beads $(0.5 \mathrm{~mm})$ and the cells were grinded with hand-powered electrical motor (VWR). Subsequently, the crude extracts were mixed with an equal volume of ethyl acetate $(\mathrm{v} / \mathrm{v})$, followed by vortex at room temperature for 2 hours. Organic and inorganic layers were separated by centrifugation at $12000 \mathrm{rpm}$ for $10 \mathrm{~min}$. Samples were extracted three times. The combined organic layers were evaporated in a vacuum oven $\left(50^{\circ} \mathrm{C}\right)$ and the remainders were resuspended in $0.5 \mathrm{~mL} 100 \%$ methanol. Then, $100 \mathrm{uL}$ of the sample were gently transferred into a HPLC vial insert and $5 \mathrm{uL}$ were injected into HPLC for OLA quantification. Under this condition, the retention time for OLA is $10.8 \mathrm{~min}$.

Olivetolic acids standards and samples were also characterized by the Perkin Elmer QSight LX50 UHPLC (ultra-high performace liquid chromatography) with the QSight 210 Mass Spectrometer under negative ESI (electrospray ionization) mode. The Agilent Zorbax Eclipse XDB-C18 $2.1 \times$ 50mm, $1.8 \mu \mathrm{m}$ (P/N 981757-902) column was used for the UHPLC system. Under the UHPLC-MS system, the retention time for olivetolic acid is $1.8 \mathrm{~min}$ and the characteristic mass/charge $\left(\mathrm{m} / \mathrm{z}\right.$ ) ratio for OLA is 223.5 and 179.5 (decarboxylated- $\mathrm{CO}_{2}$ fragment). A detailed UPLC-MS report for OLA could be found in the supplementary information.

\section{Results and Discussion}

\subsection{Biosynthesis of olivetolic acid in engineered Y. lipolytica}

Olivetolic acid (OLA) biosynthesis involves the condensation of one hexanoyl-CoA with three malonyl-CoAs by a type III PKS (tetraketide synthase) OLA synthase (C. sativa OLS; CsOLS) and an OLA cyclase (CsOAC) (Figure 1), both enzymes were derived from the Cannabis species. Previous studies have achieved trace amount of OLA production in both $E$. coli and S. cerevisiae ${ }^{21,22}$. To produce OLA in Y. lipolytica, the plasmid PYLXP'-CSOLS-CSOAC encoding the codon-optimized CSOLS and CSOAC was transformed into Y. lipolytica. Under HPLC characterization, OLA has a characteristic retention time of $10.8 \mathrm{~min}$ (Figure 2a). The resulting strain YL101 only produced $0.11 \mathrm{mg} / \mathrm{L}$ OLA after $96 \mathrm{~h}$ cultivation (Figure 2b), which 
is comparable to the initial OLA production in S. cerevisiae.

Evidence showed that some undesired byproducts could also be formed in the biosynthetic pathway of OLA ${ }^{21,22}$. We hypothesized that fusing CSOLS with CSOAC may place the two enzymes in close proximity and minimize the dissipation of intermediates. In such a way, fused OLS-OAC may efficiently transfer the intermediates to form OLA with minimal byproducts. We fused the two proteins with an amino acid linker (10xGlycine) in two different orientations. The results showed that when CSOLS was fused to the $\mathrm{N}$-terminus or C-terminus of the CSOAC, the obtained strains YL102 and YL103 showed a slightly decline in the production of OLA compared with the control strains YL101 (Figure 2c). It is possible that the fusion of these two proteins may negatively impact their correct folding and catalytic functions. Because the fusion of CSOLS with CSOAC could not further improve OLA production, the best production strain, YL101, was subjected to further engineering.

\subsection{Screening enzymes to overcome the limitation of precursor hexanoyl-CoA supply}

One of the critical challenges to improve OLA biosynthesis is the pool size of hexanoyl-CoA, which was proven to be a rate-limiting factor inside both $E$. coli and $S$. cerevisiae ${ }^{21,22}$. An acyl activating enzyme encoded by CSAAE1 from Cannabis sativa was characterized to catalyze the formation of hexanoyl-CoA from hexanoic acid, and the expression of CSAAE1 has been shown to successfully increase the titer of OLA by twofold in S. cerevisiae with the feeding of $1 \mathrm{mM}$ hexanoic acid ${ }^{22}$. Hexanoic acid is toxic to cell growth and inhibit enzyme activity. The dosage of hexanoic acid should be investigated to minimize its negative impact on cell fitness and OLA production ${ }^{21}$. Thus, CSAAE1 was expressed in conjunction with CSOLS and CSOAC in Y. lipolytica supplied with $0.5 \mathrm{mM}, 1 \mathrm{mM}$, and $2 \mathrm{mM}$ hexanoic acid at $24 \mathrm{~h}$, respectively. However, we found that there was a decline in the titer of OLA with the overexpression of CSAAE1 under these conditions when compared to the control strain with no supplementation of hexanoic acid (Figure $3 a$ ). The $\mathrm{OD}_{600}$ values tested in the process of fermentation showed that the yeast cells grew slowly with supplementation of hexanoic acid at $24 \mathrm{~h}$ and their $\mathrm{OD}_{600}$ values were lower than that of the control strain at $96 \mathrm{~h}$ (Figure $3 \mathrm{~b}$ ). This was probably due to the toxicity of hexanoic acid to $\mathrm{Y}$. lipolytica cells. We speculated that only when there were enough yeast cells with CSAAE1 
overexpression, hexanoic acid could be efficiently converted to hexanoyl-CoA, and thus its toxicity to cells could be minimized. Thus, different dosages of hexanoic acid were fed at 48 h. It was found that the titer of OLA was highest with supplementation of $0.5 \mathrm{mM}$ hexanoic acid at $48 \mathrm{~h}$ and slightly increased to $0.13 \mathrm{mg} / \mathrm{L}$ (Figure 3a), and the yeast cells didn't have observable changes in the $\mathrm{OD}_{600}$ values of the control strain (Figure $3 \mathrm{c}$ ). Thus, the supplementation of $0.5 \mathrm{mM}$ hexanoic acid at 48 hours was used for further strain screening.

Nevertheless, CSAAE1 showed lower catalytic capacity in Y. lipolytica than in S. cerevisiae $^{22}$. In order to optimize the conversion of hexanoic acid to hexanoyl-CoA, we screened a panel of six enzymes that have been annotated with acyl-CoA synthase activity. CSAAE3 encoding a peroxisomal enzyme can accept a variety of fatty acid as substrates including hexanoic acid. We next removed the C-terminal peroxisome targeting sequence 1 (PTS1) and overexpressed the codon-optimized CSAAE3 in Y. lipolytica ${ }^{23-25}$. Both the short-chain and long-chain fatty acyl-CoA synthetases, EcFadK and EcFadD from E. coli, have been shown to exhibit a catalytic activity on C6-C8 fatty acids ${ }^{21}$. The medium-chain fatty acyl-CoA synthetase SCFAA2 from S. cerevisiae and the $Y$. lipolytica native fatty acyl-CoA synthetase encoded by yIFAA1 (YALIOD17864g) were also tested in this study. The IvaE gene of Pseudomonas putida KT2440 (PpLvaE) encodes an enzyme that is active with C4-C6 carboxylic acids, including hexanoic acid ${ }^{26}$. Among these chosen hexanoyl-CoA synthetases, we found the overexpression of PplvaE in combination with CSOLS and CSOAC (strain YL110) resulted in the highest OLA production, which showed an eightfold increase in OLA titer $(1.07 \mathrm{mg} / \mathrm{L})$ (Figure 4a). P. putida has been reported to exhibit better solvent tolerance and grow under a number of harsh or hydrophobic conditions. We believe that the source of hexanoic acyl-CoA synthase played a major role to unlock the rate-limiting reactions of the initial steps of the cannabinoids pathway. Future enzymology study or directed evolution of PplvaE may further improve its catalytic function.

\subsection{Enhancing the availability of precursor malonyl-CoA and controlling $\mathrm{pH}$ to improve olivetolic acid production}

Malonyl-CoA has been reported as the major rate-limiting precursors for polyketides synthesis ${ }^{27}$. Next, we used two strategies to improve malonyl-CoA supply. In the first 
strategy, two endogenous genes yIDGA1 (YALIOE32769g) and yIDGA2 (YALIOD07986g) encoding diacylglycerol acyltransferases were knocked out, in such way, we could minimize the formation of triglycerides by blocking the acyltransferase activity of DGA1 and DGA2 ${ }^{28,29}$. Increased acyl-CoAs will reduce fatty acids synthase activity and boost the intracellular malonyl-CoA. The plasmid pYLXP'-CSOLS-CSOAC-PpLvaE was transformed into the strain YL111 (yIDGA2 knockout strain) and YL112 (yIDGA1 and $y I D G A 2$ double knockout strain), respectively. Surprisingly, the resulting strains YL113 and YL114 showed a dramatic decline in OLA production, which was contractionary to our expectation (Figure 4b). We speculate that that the increased acyl-CoAs, or acyl-ACPs may strongly feedback inhibit the activity of the acetyl-CoA carboxylase ${ }^{30,31}$, which has been reported in both prokaryotic and eukaryotic cells.

In the second strategy, the endogenous gene ylACC1 (YALIOC11407g) encoding acetyl-CoA carboxylase was overexpressed to facilitate the conversion of acetyl-CoA to malonyl-CoA ${ }^{32}$. To minimize spliceosomal or transcriptional level regulation, the two internal introns of the native gene ylACC1 were removed. The engineered strain YL115 with overexpression of ylACC1 produced $1.9 \mathrm{mg} / \mathrm{L}$ of OLA, with a 1.8-fold improvement (Figure 4b). Additionally, snf1 has been reported as the protein level regulator for ACC activity. The phosphorylation of the serine groups on ACC will strongly inhibit ACC activity ${ }^{33,34}$. For example, a snf1-resistant version of ACC mutant $\left(S C A C C 1^{5659 A, S 1157 A}\right)$ has been reported to boost intracellular malonyl-CoA level and improve 3-hydroxyl propionate or fatty acids production in S. cerevisiae ${ }^{35}$. Thus, a mutant version of $y \mid A C C 1^{\text {S667A, S1178A }}$ was obtained after alignment with amino acid sequences of SCACC1. However, the result showed that the OLA production failed to achieve improvement by overexpressing $y \mid A C C 1^{\text {S667A, S1178A }}$ (strain YL116, Figure 4b). We predict that the phosphorylation modification of ACC1 in Y. lipolytica may subject to some hidden layer of regulations, yet to be explored.

We also observed that the $\mathrm{pH}$ of the fermentation media dramatically dropped to below 3.5 during the fermentation process, due to the accumulation of organic acids. This decreased $\mathrm{pH}$ or increased proton concentration may negatively affect membrane permeability and strain physiology ${ }^{32,36}$. We next sought to control the medium pH by using 
either PBS buffer or $\mathrm{CaCO}_{3}{ }^{36}$. Supplementation of $20 \mathrm{~g} / \mathrm{L} \mathrm{CaCO}_{3}$ maintained stable $\mathrm{pH}$ and increased OLA titer by threefold, reaching $5.86 \mathrm{mg} / \mathrm{L}$ at $96 \mathrm{~h}$, whereas PBS failed to improve OLA production (Figure 5a). We speculate that the use of PBS buffer may shift the cell metabolism, for example, the cell may increase the phospholipid or cell membrane synthesis 37 , which is competing with our goal to synthesize OLA because both pathways share the same precursor malonyl-CoA.

\subsection{Debottlenecking acetyl-CoA, ATP and NADPH supply to improve olivetolic acid production}

Acetyl-CoA served as the basic building block for both hexanoyl-CoA and malonyl-CoA, and the biosynthesis of OLA from acetyl-CoA requires extensive consumption of ATP and NADPH as cofactors to facilitate the activity of ACC, hexanoyl-CoA synthase and olivetolic acid synthase ${ }^{21,22,38}$. To simplify genetic manipulations and increase strain stability, we next integrated the relevant genes carried by the plasmid pURLB-CSOLS-CSOAC-PpLvaE-yIACC1 at the genomic locus of YALIOC05907g, which was screened as an orthogonal integration site for polyunsaturated fatty acid production in Y. lipolytica ${ }^{39}$. The integrated strain YL117 yielded about $3.26 \mathrm{mg} / \mathrm{L}$ OLA in YPD complex media, which was lower than the production level $(5.86 \mathrm{mg} / \mathrm{L})$ of the strain YL115 with chemically-defined complete synthetic media (CSM-leu), possibly due to the altered gene expression profile resulting from genomic integration or shifting of culture media from CSM to YPD (Figure 5b). To probe the effect, YL117 strain was transformed with the empty plasmid pYLXP' and fermented in CSM-leu media, but the OLA titer decreased to $2.99 \mathrm{mg} / \mathrm{L}$, which indicates YPD media was better for the integrated strain (Figure 5b). Subsequently, the genomic locus of ku70 and the pBR docking site ${ }^{16}$ were chosen as integration site for comparison with the genomic locus of YALIOC05907g, resulting in the integrated strains YL118 and YL119, respectively. The strain YL118 in YPD media produced a comparatively higher OLA titer of $3.54 \mathrm{mg} / \mathrm{L}$ (Figure 11). By comparisons of the OLA titer from the integrated strain, we speculated that the supply of acetyl-CoA may create a major bottleneck for OLA synthesis in Y. lipolytica.

To improve acetyl-CoA supply, the Y. lipolytica peroxisomal matrix protein Pex10 
$(y / \mathrm{Pex} 10)^{40}$, Salmonella enterica acetyl-CoA synthetase mutant $\left(\operatorname{SeAcs}^{\mathrm{L641 \textrm {P }}}\right)^{41}$, and E. coli pyruvate dehydrogenase complex $(E C P D H)$ with the lipoate-protein ligase $A(E C L P \mid A)^{32}$ were reported to contribute to boost the availability of acetyl-CoA in Y. lipolytica or S. cerevisiae. Thus, we overexpressed these enzymes and their combination in the strain YL118. The resultant strains improved the OLA production to different extents, with the strain YL125 overexpressing the combination of ylPex10, SeAcs ${ }^{\mathrm{L} 44 \mathrm{P}}$ and ECPDH yielding the highest OLA titer of $7.43 \mathrm{mg} / \mathrm{L}$ (Figure $5 \mathrm{c}$ ). Ant1p, a peroxisomal adenine nucleotide transporter, is an integral protein of the peroxisomal membrane and is responsible for transferring ATP into peroxisomes for $\beta$-oxidation of medium-chain fatty acids, which will increase the rate of $\beta$-oxidation cycle and provide cytosolic acetyl-CoAs ${ }^{42}$. For example, the SCANT1 has been overexpressed in S. cerevisiae to supply ATP and enhance peroxisome functions ${ }^{43}$. Thus, we chose to overexpress the SCANT1 homologous gene yIANT1 (YALIOE03058g) encoding $Y$. lipolytica peroxisomal ATP/AMP transporter to increase ATP supply and the peroxisomal $\beta$-oxidation rate. The native gene yIMAE1 (YALIOE18634g) encoding malic enzyme (MAE) and MCMAE2 from Mucor circinelloides were reported to provide NADPH in Y. lipolytica ${ }^{32,44}$. We next transformed the plasmid pYLXP'-yIMAE1-yIANT1-MCMAE2 into the strain YL118 to improve the supply for both ATP and NADPH. The resultant strain YL126 produced $6.21 \mathrm{mg} / \mathrm{L}$ of OLA (Figure 5c). Next, the expression plasmid containing seven genes which overcome precursor limitations for acetyl-COA, NADPHs and ATPs were constructed and then transformed into YL118 for coexpression. The resulting strain YL127 further enhanced OLA production to $8.23 \mathrm{mg} / \mathrm{L}$ (Figure $5 \mathrm{c}$ ). Finally, the linearized gene fragments containing pYLXP'-yIPEX10-SeACS ${ }^{1641 P}$-ECPDH-ECLPIA-yIMAE1-yIANT1-MCMAE2 was integrated at the pBR docking site of the strain YL118. The engineered strain YL128 cultured in YPD media produced OLA with a titer of $9.18 \mathrm{mg} / \mathrm{L}$ (Figure 5c), which was improved by 83 times as compared to the OLA titer produced by the initial strain YL101 $(0.11 \mathrm{mg} / \mathrm{L})$. The current OLA production titer represents a threefold higher than the reported OLA titer produced in engineered S. cerevisiae in the shaker flasks ${ }^{22}$.

\section{Conclusions}


By estimation, more than $75 \%$ of the essential drugs are derived from plant secondary metabolites. In their native hosts, the active ingredients of natural products are in extremely low concentration (most often $<0.01 \%$ ) and subject to environmental, seasonal, and regional variations ${ }^{45}$. Current plant breeding and agricultural technologies cannot meet the market demand. Total chemical synthesis is not economical feasible because of the structural complexity and existence of chemical analogs. Engineering plant biosynthetic pathways in promising microbial hosts offers significant promise for scalable synthesis of essential drug components.

In this study, we have systematically investigated the enzymatic bottlenecks that restrain the efficient biosynthesis of CBD precursor olivetolic acid in the oleaginous yeast $Y$. lipolytica. We took a reverse engineering approach and sequentially identified that the supply of hexanoyl-CoA, malonyl-CoA, acetyl-COA, NADPH and ATP are the rate-limiting steps. To overcome these limitations, we have screened enzymes aiming to debottleneck the pathway limitations and redirect the carbon flux toward the end-product olivetolic acid. We discovered that the use of the $P$. putia IvaE encoding a novel acyl-CoA synthase could efficiently convert exogenously-added hexanoic acid to hexanoyl-CoA. The co-expression of the acetyl-CoA carboxylase, the pyruvate dehydrogenase bypass, the NADPH-generating malic enzyme, as well as the activation of peroxisomal $\beta$-oxidation pathway and ATP export pathway were efficient strategies to remove the pathway bottlenecks. Collectively, these strategies have led us to construct a Y. lipolytica strain that produced olivetolic acid at a titer 83 -fold higher than our initial strain $(0.11 \mathrm{mg} / \mathrm{L})$. While the current production level is low, we expect these strategies may serve as a baseline for other metabolic engineers who are interested in engineering CBD biosynthesis in oleaginous yeast species.

\section{Acknowledgement}

This project is supported by the Bill \& Melinda Gates Foundation Award (grant number OPP 1188443) and National Science Foundation (CBET-1805139). We thank Dr. Joshua Wilhide and Dr. LaCourse to help run the LC-MS characterization of OLA at the UMBC Molecular Characterization and Analysis Complex center (UMBC chemistry facility) 
and help prepare the LC-MS report.

\section{Conflicts of interests}

None declared.

\section{Author contributions}

PX designed the study, analyzed the data and revised the manuscript. J.M. performed the genetic engineering, enzyme screening and cell cultivation with input from YG. J.M wrote the manuscript and analyzed the data.

\section{References}

1. Mackie, K., Cannabinoid Receptors: Where They are and What They do. Journal of Neuroendocrinology 2008, 20 (s1), $10-14$.

2. Zou, S.; Kumar, U., Cannabinoid Receptors and the Endocannabinoid System: Signaling and Function in the Central Nervous System. International Journal of Molecular Sciences 2018, 19 (3).

3. Rosenberg, E. C.; Tsien, R. W.; Whalley, B. J.; Devinsky, O., Cannabinoids and Epilepsy. Neurotherapeutics 2015, 12 (4), 747-768.

4. Giacoppo, S.; Mandolino, G.; Galuppo, M.; Bramanti, P.; Mazzon, E., Cannabinoids: New Promising Agents in the Treatment of Neurological Diseases. Molecules 2014, 19 (11).

5. Deguchi, M.; Kane, S.; Potlakayala, S.; George, H.; Proano, R.; Sheri, V.; Curtis, W. R.; Rudrabhatla, S., Metabolic Engineering Strategies of Industrial Hemp (Cannabis sativa L.): A Brief Review of the Advances and Challenges. Frontiers in Plant Science 2020, 11, 1898.

6. Gu, Y.; Xu, P., Synthetic yeast brews neuroactive compounds. Nature Chemical Biology 2021, 17 (1), 8-9.

7. Yadav, V. G.; De Mey, M.; Giaw Lim, C.; Kumaran Ajikumar, P.; Stephanopoulos, G., The future of metabolic engineering and synthetic biology: Towards a systematic practice. Metabolic Engineering 2012, 14 (3), $233-241$.

8. Kim, D. I.; Chae, T. U.; Kim, H. U.; Jang, W. D.; Lee, S. Y., Microbial production of multiple short-chain primary amines via retrobiosynthesis. Nature Communications 2021, 12 (1), 173.

9. Banerjee, D.; Eng, T.; Lau, A. K.; Sasaki, Y.; Wang, B.; Chen, Y.; Prahl, J.-P.; Singan, V. R.; Herbert, R. A.; Liu, Y.; Tanjore, D.; Petzold, C. J.; Keasling, J. D.; Mukhopadhyay, A., Genome-scale metabolic rewiring improves titers rates and yields of the non-native product indigoidine at scale. Nature Communications 2020, $11(1), 5385$.

10. Ma, J.; Gu, Y.; Xu, P., A roadmap to engineering antiviral natural products synthesis in microbes. Current Opinion in Biotechnology 2020, 66, 140-149.

11. Ma, J.; Gu, Y.; Marsafari, M.; Xu, P., Synthetic biology, systems biology, and metabolic engineering of Yarrowia lipolytica toward a sustainable biorefinery platform. Journal of Industrial Microbiology and Biotechnology 2020, 47 (9-10), 845-862.

12. Sáez-Sáez, J.; Wang, G.; Marella, E. R.; Sudarsan, S.; Cernuda Pastor, M.; Borodina, I., Engineering the oleaginous yeast Yarrowia lipolytica for high-level resveratrol production. Metabolic Engineering 2020, 62, 51-61.

13. Liu, H.; Wang, F.; Deng, L.; Xu, P., Genetic and bioprocess engineering to improve squalene production in Yarrowia lipolytica. Bioresource Technology 2020, 317, 123991. 
14. Palmer, C. M.; Miller, K. K.; Nguyen, A.; Alper, H. S., Engineering 4-coumaroyl-CoA derived polyketide production in Yarrowia lipolytica through a $\beta$-oxidation mediated strategy. Metabolic Engineering 2020, 57, 174-181.

15. Marsafari, M.; Xu, P., Debottlenecking mevalonate pathway for antimalarial drug precursor amorphadiene biosynthesis in Yarrowia lipolytica. Metabolic Engineering Communications 2020, 10, e00121.

16. Gu, Y.; Ma, J. B.; Zhu, Y. L.; Ding, X. Y.; Xu, P., Engineering Yarrowia lipolytica as a Chassis for De Novo Synthesis of Five Aromatic-Derived Natural Products and Chemicals. Acs Synthetic Biology 2020, 9 (8), 2096-2106.

17. Luo, X.; Reiter, M. A.; d'Espaux, L.; Wong, J.; Denby, C. M.; Lechner, A.; Zhang, Y.; Grzybowski, A. T.; Harth, S.; Lin, W.; Lee, H.; Yu, C.; Shin, J.; Deng, K.; Benites, V. T.; Wang, G.; Baidoo, E. E. K.; Chen, Y.; Dev, I.; Petzold, C. J.; Keasling, J. D., Complete biosynthesis of cannabinoids and their unnatural analogues in yeast. Nature 2019, 567 (7746), 123-126.

18. Gietz, R. D.; Woods, R. A., Transformation of yeast by lithium acetate/single-stranded carrier DNA/polyethylene glycol method. Methods Enzymol 2002, 350, 87-96.

19. Wong, L.; Engel, J.; Jin, E.; Holdridge, B.; Xu, P., YaliBricks, a versatile genetic toolkit for streamlined and rapid pathway engineering in Yarrowia lipolytica. Metab Eng Commun 2017, 5, 68-77.

20. Fickers, P.; Le Dall, M. T.; Gaillardin, C.; Thonart, P.; Nicaud, J. M., New disruption cassettes for rapid gene disruption and marker rescue in the yeast Yarrowia lipolytica. J Microbiol Meth 2003, 55 (3), 727-737.

21. Tan, Z.; Clomburg, J. M.; Gonzalez, R., Synthetic Pathway for the Production of Olivetolic Acid in Escherichia coli. ACS Synth Biol 2018, 7 (8), 1886-1896.

22. Luo, X. Z.; Reiter, M. A.; d'Espaux, L.; Wong, J.; Denby, C. M.; Lechner, A.; Zhang, Y. F.; Grzybowski, A. T.; Harth, S.; Lin, W. Y.; Lee, H.; Yu, C. H.; Shin, J.; Deng, K.; Benites, V. T.; Wang, G.; Baidoo, E. E. K.; Chen, Y.; Dev, I.; Petzold, C. J.; Keasling, J. D., Complete biosynthesis of cannabinoids and their unnatural analogues in yeast. Nature 2019, 567 (7746), 123-+.

23. Stout, J. M.; Boubakir, Z.; Ambrose, S. J.; Purves, R. W.; Page, J. E., The hexanoyl-CoA precursor for cannabinoid biosynthesis is formed by an acyl-activating enzyme in Cannabis sativa trichomes. Plant J 2012, 71 (3), 353-365.

24. Emanuelsson, O.; Brunak, S.; von Heijne, G.; Nielsen, H., Locating proteins in the cell using TargetP, SignalP and related tools. Nat Protoc 2007, 2 (4), 953-971.

25. Emanuelsson, O.; Nielsen, H.; Von Heijne, G., ChloroP, a neural network-based method for predicting chloroplast transit peptides and their cleavage sites. Protein Sci 1999, 8 (5), 978-984.

26. Rand, J. M.; Pisithkul, T.; Clark, R. L.; Thiede, J. M.; Mehrer, C. R.; Agnew, D. E.; Campbell, C. E.; Markley, A. L.; Price, M. N.; Ray, J.; Wetmore, K. M.; Suh, Y.; Arkin, A. P.; Deutschbauer, A. M.; Amador-Noguez, D.; Pfleger, B. F., A metabolic pathway for catabolizing levulinic acid in bacteria. Nat Microbiol 2017, 2 (12), 1624-1634.

27. Yang, D.; Kim, W. J.; Yoo, S. M.; Choi, J. H.; Ha, S. H.; Lee, M. H.; Lee, S. Y., Repurposing type III polyketide synthase as a malonyl-CoA biosensor for metabolic engineering in bacteria. Proceedings of the National Academy of Sciences 2018, 115 (40), 9835.

28. Gu, Y.; Ma, J.; Zhu, Y.; Xu, P., Refactoring Ehrlich Pathway for High-Yield 2-Phenylethanol Production in Yarrowia lipolytica. ACS Synth Biol 2020, 9 (3), 623-633.

29. Silverman, A. M.; Qiao, K.; Xu, P.; Stephanopoulos, G., Functional overexpression and characterization of lipogenesis-related genes in the oleaginous yeast Yarrowia lipolytica. Appl Microbiol Biotechnol 2016, 100 (8), 3781-98.

30. Andre, C.; Haslam, R. P.; Shanklin, J., Feedback regulation of plastidic acetyl-CoA carboxylase by 18:1-acyl 
carrier protein in <em>Brassica napus</em>. Proceedings of the National Academy of Sciences 2012, 109 (25), 10107.

31. Davis Mark, S.; Cronan John, E., Inhibition of Escherichia coliAcetyl Coenzyme A Carboxylase by Acyl-Acyl Carrier Protein. Journal of Bacteriology 2001, 183 (4), 1499-1503.

32. Liu, H.; Marsafari, M.; Wang, F.; Deng, L.; Xu, P., Engineering acetyl-CoA metabolic shortcut for eco-friendly production of polyketides triacetic acid lactone in Yarrowia lipolytica. Metab Eng 2019, 56, 60-68.

33. Shi, S.; Chen, Y.; Siewers, V.; Nielsen, J., Improving Production of Malonyl Coenzyme A-Derived Metabolites by Abolishing Snf1-Dependent Regulation of Acc1. mBio 2014, 5 (3).

34. Shirra, M. K.; Patton-Vogt, J.; Ulrich, A.; Liuta-Tehlivets, O.; Kohlwein, S. D.; Henry, S. A.; Arndt, K. M., Inhibition of Acetyl Coenzyme A Carboxylase Activity Restores Expression of the <em $>\mid N 01</$ em $>$ Gene in a <em>snf1</em>Mutant Strain of <em>Saccharomyces cerevisiae</em>. Molecular and Cellular Biology 2001, 21 (17), 5710.

35. Yuan, S. F.; Yi, X.; Johnston, T. G.; Alper, H. S., De novo resveratrol production through modular engineering of anEscherichia coli-Saccharomyces cerevisiaeco-culture. Microb Cell Fact 2020, 19 (1).

36. LV, Y.; Marsafari, M.; Koffas, M.; Zhou, J.; Xu, P., Optimizing Oleaginous Yeast Cell Factories for Flavonoids and Hydroxylated Flavonoids Biosynthesis. ACS Synth Biol 2019, 8 (11), 2514-2523.

37. Perli, G.; Pessoa, A. C. S. N.; Balbino, T. A.; de la Torre, L. G., lonic strength for tailoring the synthesis of monomodal stealth cationic liposomes in microfluidic devices. Colloids and Surfaces B: Biointerfaces 2019, 179, 233-241.

38. Xu, P.; Qiao, K.; Ahn, W. S.; Stephanopoulos, G., Engineering Yarrowia lipolytica as a platform for synthesis of drop-in transportation fuels and oleochemicals. Proceedings of the National Academy of Sciences 2016, 113 (39), 10848-10853.

39. Gemperlein, K.; Dietrich, D.; Kohlstedt, M.; Zipf, G.; Bernauer, H. S.; Wittmann, C.; Wenzel, S. C.; Muller, R., Polyunsaturated fatty acid production by Yarrowia lipolytica employing designed myxobacterial PUFA synthases. Nat Commun 2019, 10.

40. Markham, K. A.; Palmer, C. M.; Chwatko, M.; Wagner, J. M.; Murray, C.; Vazquez, S.; Swaminathan, A.; Chakravarty, I.; Lynd, N. A.; Alper, H. S., Rewiring Yarrowia lipolytica toward triacetic acid lactone for materials generation. P Natl Acad Sci USA 2018, 115 (9), 2096-2101.

41. Lian, J. Z.; Si, T.; Nair, N. U.; Zhao, H. M., Design and construction of acetyl-CoA overproducing Saccharomyces cerevisiae strains. Metab Eng 2014, 24, 139-149.

42. van Roermund, C. W. T.; Drissen, R.; van den Berg, M.; ljlst, L.; Hettema, E. H.; Tabak, H. F.; Waterham, H. R.; Wanders, R. J. A., Identification of a peroxisomal ATP carrier required for medium-chain fatty acid beta-oxidation and normal peroxisome proliferation in Saccharomyces cerevisiae. Mol Cell Biol 2001, 21 (13), 4321-4329.

43. Liu, G. S.; Li, T.; Zhou, W.; Jiang, M.; Tao, X. Y.; Liu, M.; Zhao, M.; Ren, Y. H.; Gao, B.; Wang, F. Q.; Wei, D. Z., The yeast peroxisome: A dynamic storage depot and subcellular factory for squalene overproduction. Metab Eng 2020, 57, 151-161.

44. Qiao, K. J.; Wasylenko, T. M.; Zhou, K.; Xu, P.; Stephanopoulos, G., Lipid production in Yarrowia lipolytica is maximized by engineering cytosolic redox metabolism. Nat Biotechnol 2017, 35 (2), 173-177.

45. Newman, D. J.; Cragg, G. M., Natural Products as Sources of New Drugs over the Nearly Four Decades from 01/1981 to 09/2019. Journal of Natural Products 2020, 83 (3), 770-803. 


\section{Figure captions}

Figure 1. Engineered metabolic pathway for synthesis of olivetolic acid (OLA) in Y. lipolytica. ACC1, acetyl-CoA-carboxylase; ACS, acetic acid synthase; ANT1, adenine nucleotide transporter; CSOAC, OLA cyclase from Cannabis sativa; CsOLS, OLA synthase from C. sativa; DGA1 and DGA2, diacylglycerol acyltransferases; FAS, fatty acid synthase; MAE, malic enzyme; PDH, pyruvate dehydrogenase; PEX10, peroxisomal matrix protein.

Figure 2. Production of olivetolic acid (OLA) by recruiting OLS and OAC. (a) In vivo production of OLA in YL101 strain. (a) Extracts were analyzed by HPLC and signals were compared to authentic OLA standards (stds). (b) Time profiles of OLA titer in YL101 strain. (c) OLA production in engineered strains by expressing OLS and OAC or their fusions.

Figure 3. Olivetolic acid (OLA) and cell growth profile of YL104 strain expressing CSAAE1 with the supplementation of hexanoic acid (HA). (a) OLA titers in YL104 strain supplied with 0.5 $\mathrm{mM}, 1 \mathrm{mM}$, and $2 \mathrm{mM}$ hexanoic acid at $24 \mathrm{~h}$ or $48 \mathrm{~h}$. (b) Cell growth profile of YL104 strain fed with different dosages of HA at $24 \mathrm{~h}$. (c) Cell growth profile of YL104 strain fed with different dosages of $\mathrm{HA}$ at $48 \mathrm{~h}$.

Figure 4. Boosting the supply of precursors hexanoyl-CoA and malonyl-CoA to improve olivetolic acid (OLA) production. (a) OLA titers in engineered strains by expressing different hexanoyl-CoA synthetases for optimizing the conversion of hexanoic acid to hexanoyl-CoA. (b) OLA titers in engineered strains for enhancing the availability of malonyl-CoA.

Figure 5. Improve olivetolic acid (OLA) production by controlling $\mathrm{pH}$ and increasing acetyl-COA, ATP and NADPH supply. (a) OLA titers after controlling $\mathrm{pH}$ of the fermentation medium by using either PBS buffer or $\mathrm{CaCO}_{3}$. (b) OLA titers in strains of integrating plasmid into different the genomic integration sites in YPD or CSM-leu medium. (c) OLA titers in engineered strains by expressing different enzymes and their combinations for improving the supply of acetyl-CoA, ATP and NADPH. 


\section{Figures}

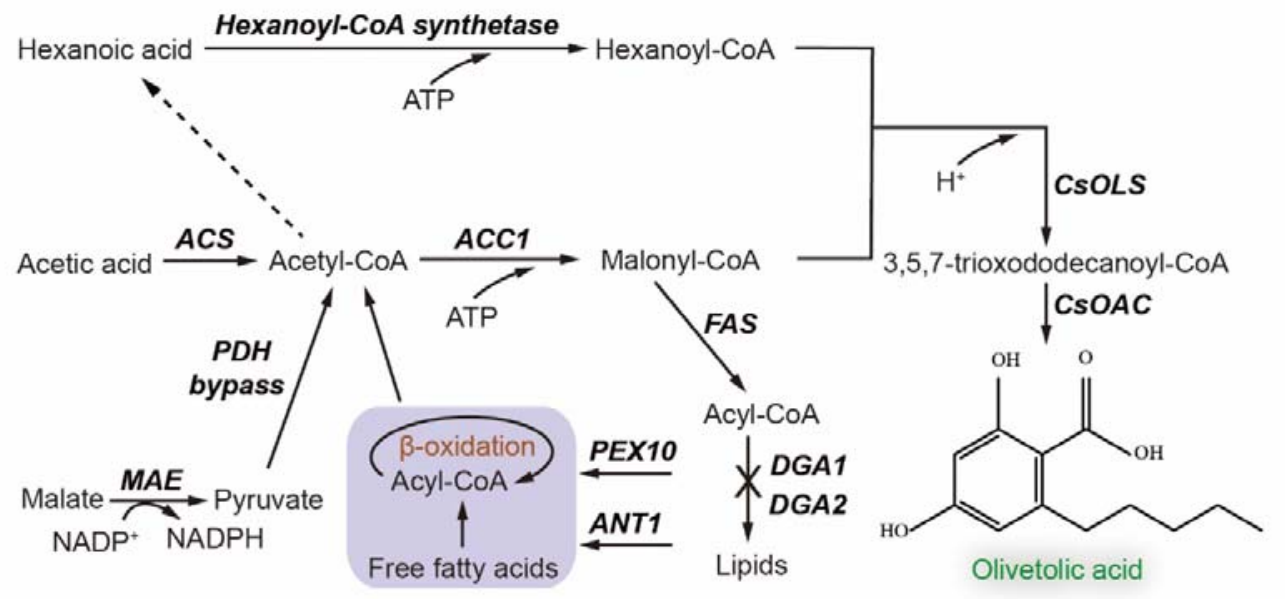

Figure 1

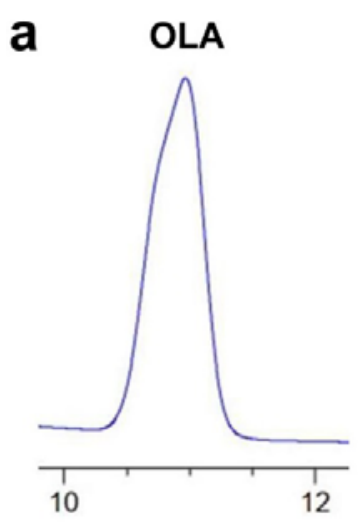

Retention time $(\min )$
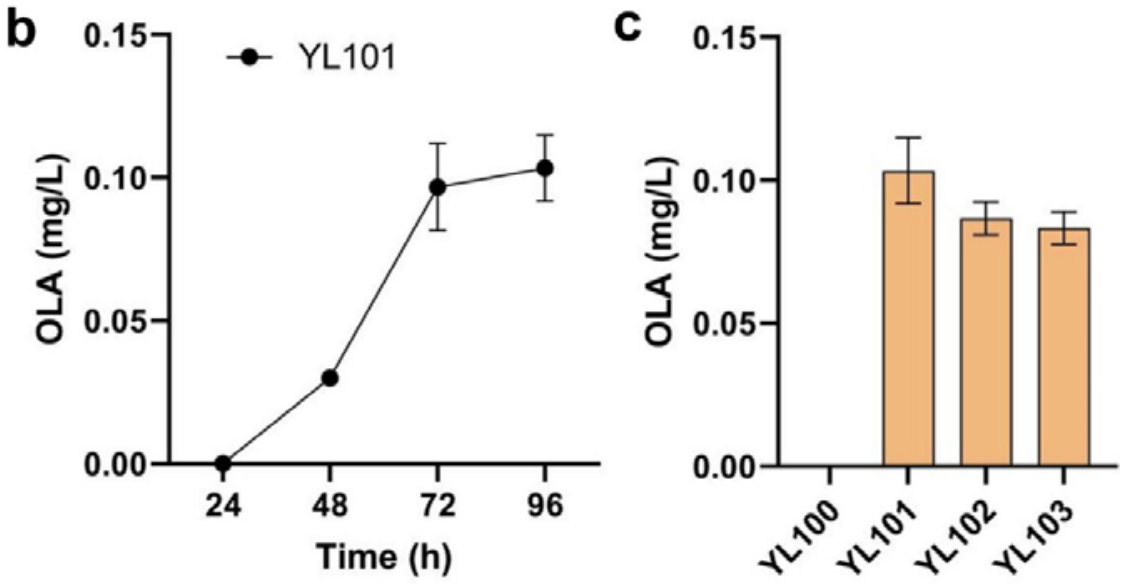

Figure 2 
bioRxiv preprint doi: https://doi.org/10.1101/2021.06.10.447928; this version posted June 10,2021. The copyright holder for this preprint (which was not certified by peer review) is the author/funder, who has granted bioRxiv a license to display the preprint in perpetuity. It is made available under aCC-BY-NC-ND 4.0 International license.
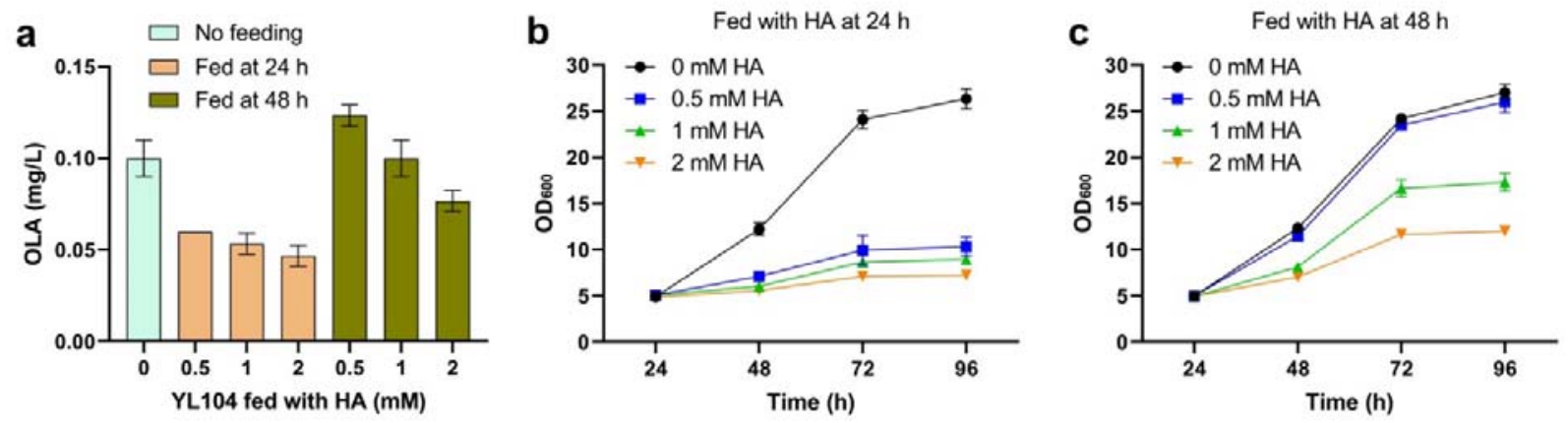

Figure 3
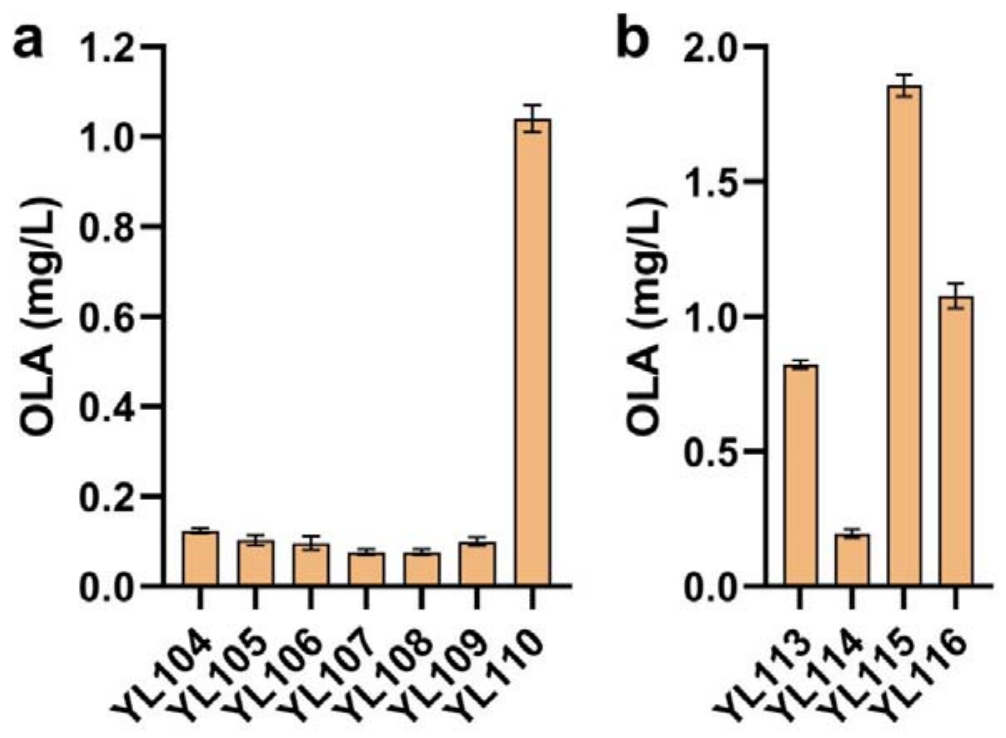

Figure 4 

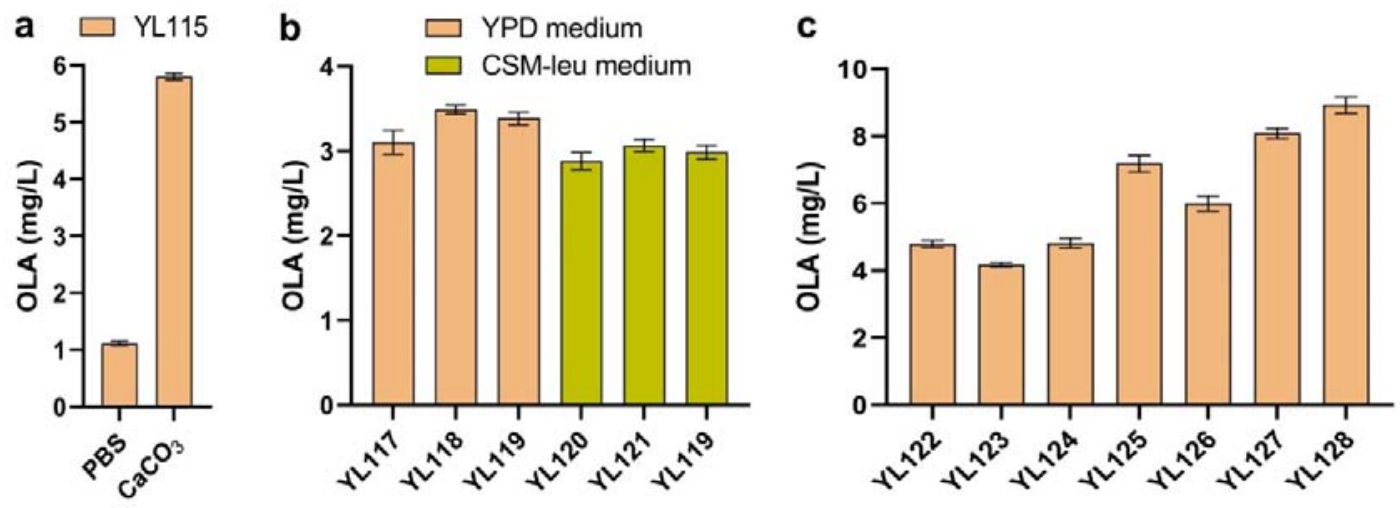

Figure 5 